\title{
OPEN Transcriptomics and metabolomics reveal the adaption of Akkermansia muciniphila to high mucin by regulating energy homeostasis
}

\author{
Xinyue Liu ${ }^{1}$, Fan Zhao ${ }^{1}$, Hui Liu ${ }^{1}$, Yunting Xie ${ }^{1}$, Di Zhao ${ }^{1}$ \& Chunbao $\mathrm{Li}^{1,2} \bowtie$
}

In gut, Akkermansia muciniphila (A. muciniphila) probably exerts its probiotic activities by the positive modulation of mucus thickness and gut barrier integrity. However, the potential mechanisms between A. muciniphila and mucin balance have not been fully elucidated. In this study, we cultured the bacterium in a $\mathrm{BHI}$ medium containing $0 \%$ to $0.5 \%$ mucin, and transcriptome and gas chromatography mass spectrometry (GC-MS) analyses were performed. We found that $0.5 \%(\mathrm{~m} / \mathrm{v})$ mucin in a $\mathrm{BHI}$ medium induced 1191 microbial genes to be differentially expressed, and 49 metabolites to be changed. The metabolites of sorbose, mannose, 2,7 -anhydro- $\beta$-sedoheptulose, fructose, phenylalanine, threonine, lysine, ornithine, asparagine, alanine and glutamic acid were decreased by $0.5 \%$ mucin, while the metabolites of leucine, valine and $\mathrm{N}$-acetylneuraminic acid were increased. The association analysis between transcriptome and metabolome revealed that $A$. muciniphila gave strong responses to energy metabolism, amino sugar and nucleotide sugar metabolism, and galactose metabolism pathways to adapt to high mucin in the medium. This finding showed that only when mucin reached a certain concentration in a BHI medium, $A$. muciniphila could respond to the culture environment significantly at the level of genes and metabolites, and changed its metabolic characteristics by altering the effect on carbohydrates and amino acids.

Akkermansia muciniphila (A. muciniphila) is a Gram-negative bacterium and belongs to the phylum Verrucomicrobia $^{1-4}$. Its abundance in the gut is largely related to age, diet and health status of the host. This bacterium begins to colonize human intestinal tract at the early life, and its abundance increases to healthy adult levels $\left(10^{8}\right.$ cells/g) within a year, but falls in old ages ${ }^{5}$. Higher abundance of $A$. muciniphila is associated with a healthier metabolic status in overweight or obese subjects ${ }^{6}$. Previous studies showed that the abundance of $A$. muciniphila is inversely related to host metabolic diseases ${ }^{7-10}$, indicating protective and anti-inflammatory potentials.

In the gut, mucin-degrading bacteria reside in the loose mucus layer, where A. muciniphila utilizes mucin as nutrient for growth ${ }^{11}$. Mucus is an important defense barrier and comprises of complex polysaccharide-proteins (i.e., mucins) with a polymeric structure, which is secreted by goblet cells in epithelial tissue. Mucus comprises of a firm inner layer and a loose outer layer. The thickness of the mucus layer increases from $125 \mu \mathrm{m}$ at the jejunum to $830 \mu \mathrm{m}$ at the colon in the gastrointestinal tract ${ }^{12,13}$. The differences in mucus thickness reflect the protective effects of the mucus. Patients with ulcerative colitis have a thinner mucus layer in the tissue, while patients with Crohn's disease have normal or even thicker mucus layer in the colon tissue ${ }^{14,15}$. Similarly, the thickness of the mucus layer is strongly related to the abundance of $A$. muciniphila in the gastrointestinal tract. Intake of arabinoxylans may increase colonic mucus thickness and the abundance of $A$. muciniphila in humanized rats ${ }^{16}$. High-fat diet would reduce the abundance of $A$. muciniphila ${ }^{17}$, while daily gavage of $A$. muciniphila can restore mucus layer thickness upon high fat $\operatorname{diet}^{18}$. In aged mice the thickness of the colonic mucus layer was reduced about sixfolds relative to young mice ${ }^{19}$. Thus, mucus alterations appear to characterize gut diseases in response to intestinal microbes (including A. muciniphila) or host-derived inflammatory mediators. However, the exact

\footnotetext{
${ }^{1}$ Key Laboratory of Meat Processing and Quality Control, Ministry of Education, Key Laboratory of Meat Processing, Ministry of Agriculture and Rural Affairs, Jiangsu Collaborative Innovation Centre of Meat Production and Processing, Quality and Safety Control, Meat Production, College of Food Science and Technology, Nanjing Agricultural University, Weigang 1\#, Nanjing 210095, People's Republic of China. ${ }^{2}$ National Center for International Research on Animal Gut Nutrition, Nanjing Agricultural University, Nanjing 210095, People's Republic of China. ${ }^{\square}$ email: chunbao.li@njau.edu.cn
} 
mechanisms by which $A$. muciniphila exerts the beneficial impact on heath associating with mucus have not been fully elucidated.

Mucins are large extracellular glycoproteins and consist of protein backbones decorated with a variety of carbohydrate chains, which are directly correlated with the protective properties of mucus gel. The mucin protein core contains highly glycosylated regions comprising of $80 \%$ carbohydrates primarily of $\mathrm{N}$-acetylgalactosamine (GalNac), N-acetylglucosamine (GlcNac), fucose (Fuc), galactose (Gal) and N-acetylneuraminic acid (Neu5Ac) and traces of sulfate $\left(\mathrm{SO}_{4}{ }^{2-}\right)$ and mannose (Man), while the peptide backbone is rich in threonine, serine, cysteine and proline $\mathrm{e}^{20,21}$. A. muciniphila could directly utilize glycans and amino acids from the peptide backbone in mucins as a nutrient. Stable isotope labeling and in situ hybridization imaging revealed that $A$. muciniphila has the ability to use host-protein derived amino acids in vivo ${ }^{22}$. A. muciniphila exerted its metabolic properties attributed to the mucin-degrading enzymes. The whole genome of $A$. muciniphila has been sequenced, and it indicates that many genes encode mucin-degrading enzymes, including glycosidases and sulfatases ${ }^{23}$. These enzymes play an important role in mucin degradation, which release glycans for the growth of the mucin-degrading bacteria and affect other residents in the gut. Hexosaminidase hydrolyzes terminal non-reducing $\mathrm{N}$-acetyl-D-hexosamine residues in $\mathrm{N}$-acetyl-beta-D-hexosaminides ${ }^{24,25}$. The $\beta$-galactosidase hydrolyzes $\beta$-D-galactosyl residues from the non-reducing ends of glycoconjugates ${ }^{26}$. The $\alpha$-L-fucosidase liberates terminal $\alpha$-linked $L$-fucose from the oligosaccharides of various glycoconjugates including mucin glycoprotein ${ }^{27}$. The $\alpha-\mathrm{N}$-acetylglucosaminidase is involved in sulfate degradation ${ }^{28}$. The a-galactosidase hydrolyzes the terminal alpha-galactosyl moieties from glycolipids and glycoproteins ${ }^{29}$. The $\alpha$-glucosidase hydrolyzes non-reducing terminal $\alpha$-D-glucose residues with release of $\alpha$-D-glucose ${ }^{30}$.

The in vitro cultivation method has been applied to study the responses of $A$. muciniphila to different levels of mucin. Ottman et al. applied a genome-scale metabolic model to predict how A. muciniphila utilized mucinderived monosaccharides, including fucose, galactose, and $\mathrm{N}$-acetylglucosamine in a minimal growth medium ${ }^{31}$. A. muciniphila prefer to grow in a special medium (a medium containing GlcNAc or GalNAc) or a BHI medium. In a BHI medium, it is not fully understood how A. muciniphila adapts to different physiological conditions varying with mucin contents.

In this study, we integrated transcriptomic and metabolomic analyses to explore the responses of A. muciniphila to mucin addition in a BHI medium, and its underlying mechanism was interpreted. To our knowledge, this is the first study that has evaluated metabolites profiles of A. muciniphila and mucin using transcriptome and GC-MS in vitro, and it will be an intensive research for the physiology of A. muciniphila to exert its probiotic functions in gut.

\section{Results and discussion}

Mucin addition affected growth rate and morphology of $\boldsymbol{A}$. muciniphila. Bacterial growth curves indicated a mucin-dependent pattern for A. muciniphila. The absorbance changed slightly when A. muciniphila was grown in a mucin medium (Fig. 1A). This might be associated with the lack of rumen fluid in our mucin medium. Instead, this bacterium could grow better in a BHI medium alone or containing mucin. Compared with control (BHI-only), it took a shorter time for the bacterium in the BM0.5 group to reach the maximum abundance ( $35 \mathrm{~h}$ for the control group vs. $28 \mathrm{~h}$ for the BM0.5 group, Fig. 1A). The times for the BM0.05 and BM0.2 groups were $31 \mathrm{~h}$ and $30 \mathrm{~h}$, respectively. In our previous in vitro cultivation studies, two different proteins (soybean and chicken proteins) were added as additional nitrogen source for A. muciniphila growth in a $\mathrm{BHI}$ medium, and we found that the $\mathrm{OD}_{600}$ values of the chicken-protein-supplied group were higher than those of the BHI-only group ${ }^{32}$. A. muciniphila in mucin-added BHI medium had shorter time to reach the maximum abundance, confirming the findings of Ottman et al..$^{31}$ that the growth rate of A. muciniphila was increased greatly when a minimal medium was mixed with $0.25 \%$ mucin and glucose. It was notable that $0.5 \%$ mucin increased microbial absorbance in the present study and Ottman et al. ${ }^{31}$. Previous studies indicated that B. thetaiotaomicron showed a diauxic growth curve in a porcine mucin $\mathrm{O}$-glycans medium ${ }^{33}$. However, mucin addition to the BHI medium induced $A$. muciniphila to grow in a unimodal growth curve in the present study. Scanning electron microscopy showed that the size of $A$. muciniphila was smaller when it was grown in a BHI medium containing $0.5 \%$ mucin compared with control or lower levels of mucin (Fig. 1B). van der Ark also observed that $A$. muciniphila had a smaller size when growing in a basic mucin medium compared with those being cultivated in a soy protein medium ${ }^{34}$. A. muciniphila growing in the BM0.5 medium had smaller diameter than growing in the BHI-only medium (Fig. 1B). Most inclusions were considered the reserves for nutrients or energy in response to nutrient imbalance ${ }^{35}$. According to these observations, we speculate that mucin addition in BHI medium promotes the growth and division of $A$. muciniphila, as the faster the cell division rate, the smaller the cell morphology.

Transcriptomic analysis of $\boldsymbol{A}$. muciniphila in response to mucin addition in $\mathrm{BHI}$ medium. Morphological and molecular changes may occur to A. muciniphila when the medium is changed. And thus wholegenome sequencing was performed to establish a set of reference sequences for transcriptome analysis (Supplementary Fig. S1, Fig. S2, Table S1 and Table S2). Transcriptome sequencing generated 15 to 34 million raw reads per sample, and there were 18,383,453, 18,396,179, 23,347,600 and 19,791,598 clean reads in the BHI-only, BM0.05, BM0.2 and BM0.5 groups, respectively. The Q20 values (sequencing error rate $<1 \%$ ) were greater than $95.66 \%$. The average GC content of four groups were $57.94 \%, 57.43 \%, 56.39 \%$ and $51.69 \%$, respectively and the rRNA reads accounted for less than $0.35 \%$ of raw reads in the studied samples (Supplementary Table S3). These results demonstrated that the quantity and quality of sequencing data were sufficient to ensure accurate sequence assembly and adequate transcriptome coverage. 
A

B
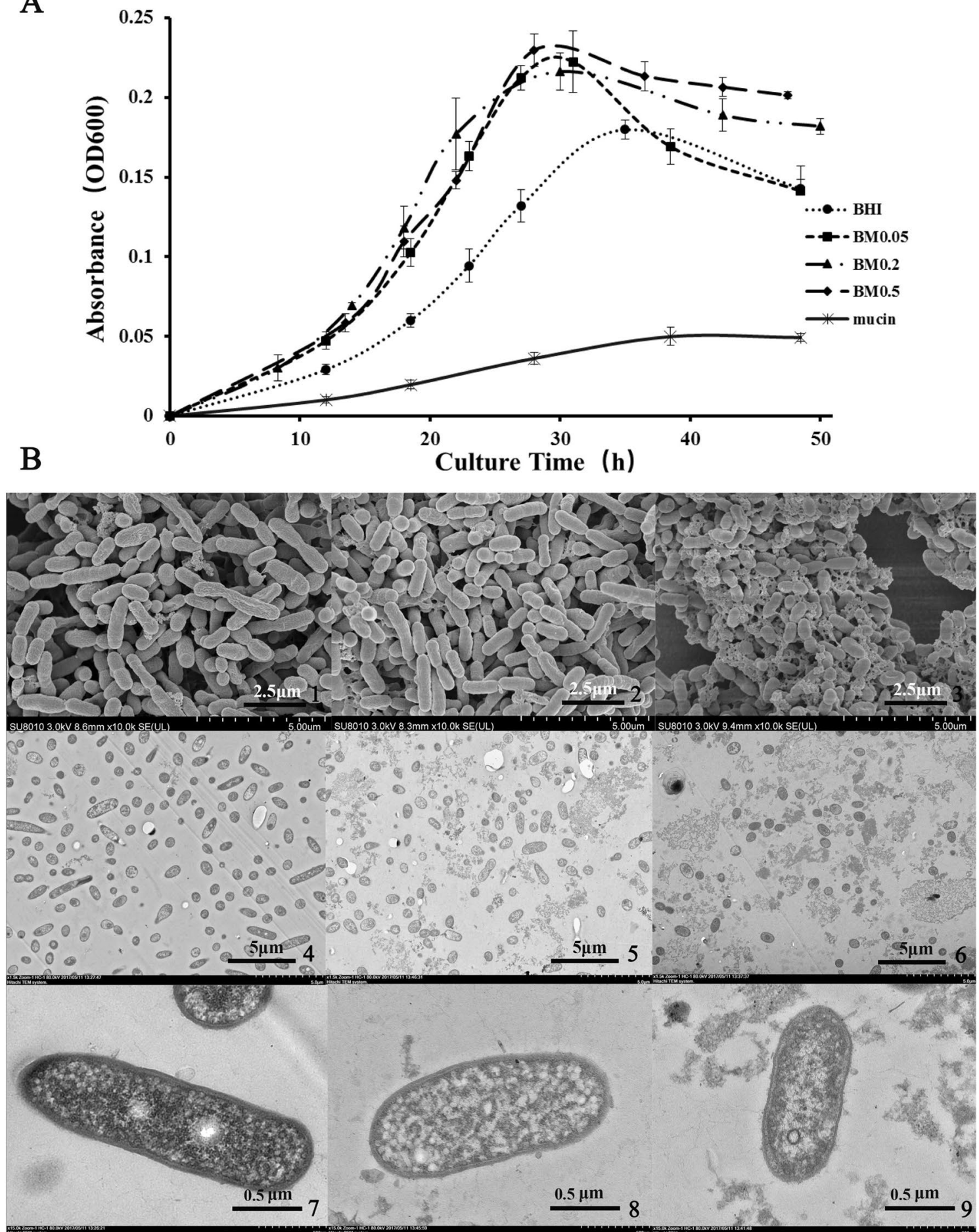

Figure 1. Mucin-dependent changes of A. muciniphila. (A) Growth curves; (B) Scanning and transmission electron microscopy images of $A$. muciniphila growing in BHI, BM0.05 and BM0.5, respectively. Among them, numbers 1, 4 and 7 represent groups without mucin; numbers 2, 5 and 8 represent groups with $0.05 \%$ mucin; numbers 3, 6 and 9 represent groups with $0.5 \%$ mucin.

Principle component analysis (PCA) of transcriptomic data revealed that the first two principal components (PCs) accounted for $91 \%$ of the total variance. The PC1 mainly reflects the differences between the control and $0.5 \%$ mucin groups, indicating a significant impact of mucin addition on the growth of A. muciniphila (Fig. 2A). Venn diagram and volcano plots indicated that higher mucin addition resulted in higher number of DEGs (Fig. 2B-E). 
A
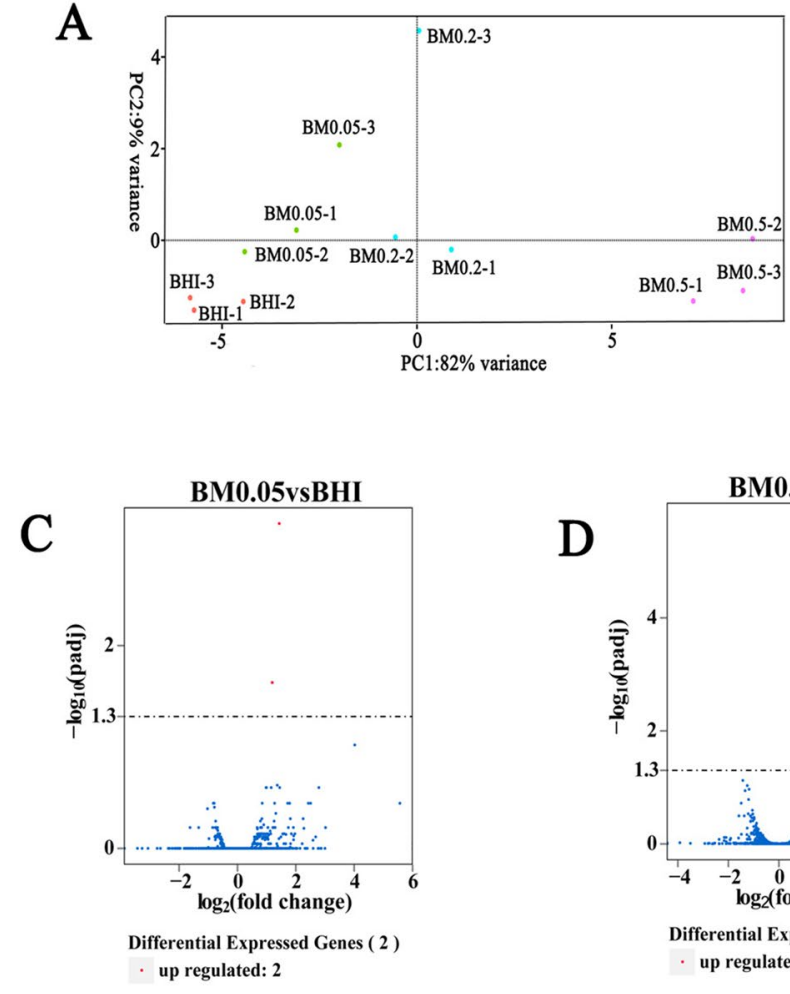

B

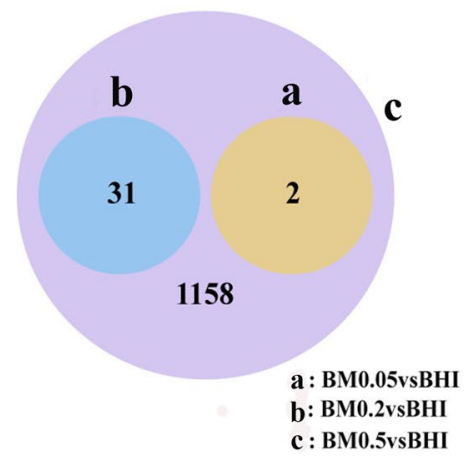

BM0.2vsBHI

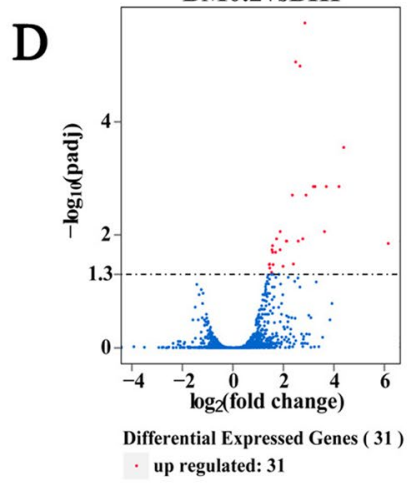

BM0.5vsBHI

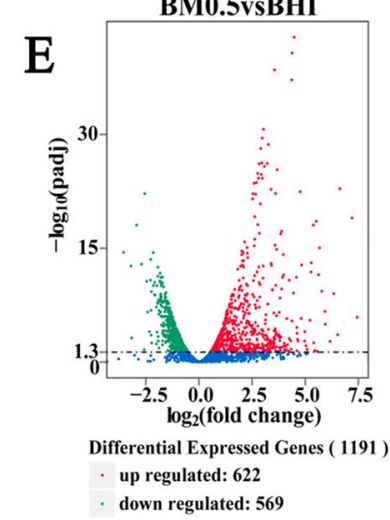

Figure 2. DEGs in A. muciniphila in response to mucin. (A) scatter plot of principal component analysis; (B) Venn diagram of DEGs; (C) Volcano plot of DEGs in BM0.05; (D) Volcano plot of DEGs in BM0.2; (E) Volcano plot of DEGs in BM0.5. Red spots represent upregulated genes, and green spots represent downregulated genes.

A

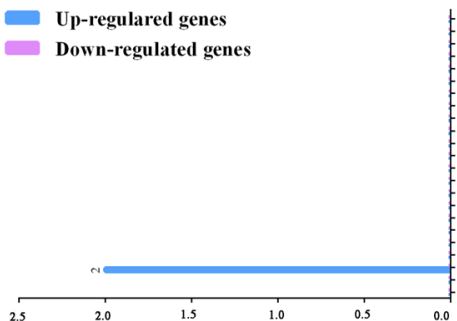

B

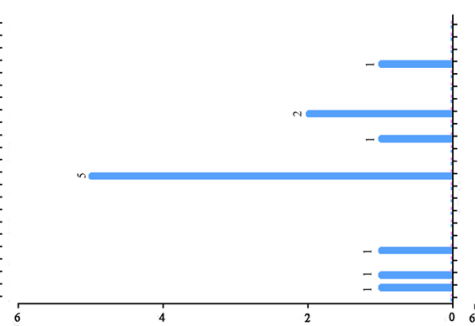

$\mathrm{C}$

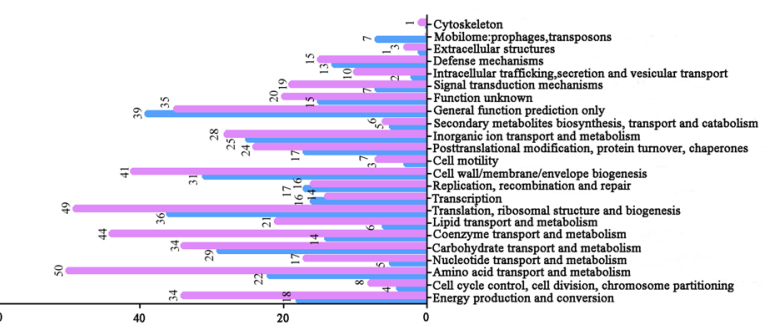

Figure 3. COG annotations of DEGs. (A) to (C) DEGs in BM0.05, BM0.2 and BM0.5 groups in the COG database, respectively.

Functional analysis of DEGs. The number of DEGs increased as mucin addition to the BHI medium increased. When $0.05 \%$ mucin was added, only two genes were upregulated involving amino acid transport and metabolism (Fig. 3A; Supplementary Fig. S3, Fig. S4A, Table S4). In the BM0.2 group, 31 DEGs were annotated to the categories of "cell wall, membrane and envelope biogenesis", "ATPase activity", and "nucleoside-triphosphatase activity" (Fig. 3B; Supplementary Fig. S3, Fig. S4B). In the BM0.5 group, 622 DEGs were upregulated and 569 DEGs were downregulated, which were primarily related to cell growth and energy metabolism. Many of the upregulated genes were indicators of increased growth ("translation, ribosomal structure and biogenesis", "cell wall, membrane and envelope biogenesis", "carbohydrate transport and metabolism", and so on) in the COG database (Fig. 3C). The top GO terms were most enriched in biological process and cell homeostasis that related to mucin degradation (Supplementary Fig. S4C). This finding indicates that $0.5 \%$ mucin can largely affect the growth and regulation of biological process in A. muciniphila. Seventeen and 12 DEGs were enriched in ribosome and glycan degradation, respectively (Supplementary Table S4), of which a gene encoding $\alpha$-amylase (Amuc_1812) was upregulated. This enzyme catalyzes the hydrolysis of $\alpha$-1,4-glucosidic bonds in starch and a-glucans ${ }^{36}$. However, Ottman et al. found that this gene was downregulated in $0.5 \%$ mucin and glucose in the minimal medium ${ }^{31}$. The difference between our study and Ottman et al. may be due to the higher glucose abun- 
dance in BHI medium ${ }^{31}$. The growth rate of bacteria is affected by many factors, e.g., the cellular machinery of ribosome, RNA polymerase activity, ATP concentration, cell wall integrity, and DNA replication ${ }^{37}$. This explains the upregulation of many DEGs in the ribosome pathway in BM0.5 group.

Transcriptomic data indicated that addition of $0.05 \%$ mucin may increase ammonia assimilation in A. muciniphila by upregulating gene expression of glutamine synthetase and glutamate synthase. Addition of $0.2 \%$ mucin may enhance ATPase, cell adhesion, glycan degradation and peptidoglycan biosynthesis. Higher mucin addition $(0.5 \%)$ upregulated gene expression of hexosaminidases (HexA_B), $\beta$-galactosidases (LacZ), $\alpha$-L-fucosidase (FucA), $\alpha$-N-acetylglucosaminidase (NaGlu), $\alpha$-galactosidase (galA) and $\alpha$-glucosidase (malZ) (Fig. 4A). However, the in vitro activities of $\beta$-hexosaminidase, $\beta$-galactosidase and $\alpha$-L-fucosidase increased when the mucin addition increased from 0 to $0.05 \%$ but the enzymatic activities decreased greatly when the mucin addition further increased (Fig. 4B-D). We speculate that the inconsistence between in vitro activities and gene expression could be attributed to related glycoside hydrolases in the BM0.5 group that could be attached to mucin and filtered out.

DEGs related to DNA replication and cell morphology were listed in heatmap (Fig. 5; Supplementary Table S5). The genes related to the faster growth were up-regulated and the genes related to smaller sizes of A. muciniphila were downregulated in the BM0.5 group. In addition, the more abundant carbon source in the BM0.5 group may also contribute to the faster growth of $A$. muciniphila. The cell shape determining protein MreB (Amuc_0540) was downregulated by fourfolds in the BM0.5 group compared with BHI-only group. The $\mathrm{mreB}$ is a key gene regulating cell morphology and increasing cell length in A. muciniphila ${ }^{34}$.

Metabolome profiles of $\boldsymbol{A}$. muciniphila in response to mucin addition to $\mathrm{BHI}$ medium. To explore the metabolome profiles of A. muciniphila when different amounts of mucin were added to the BHI medium, the BHI, BM0.05 and BM0.5 cultures were subject to GC-MS non-target metabolic analysis. An OPLS-DA model showed a good separation of metabolites among the three groups (Supplementary Fig. S5 and Table S6).

A total of 161 compounds were identified and analysis of variance showed that 49 compounds of them were significantly different $(\mathrm{P}<0.05$, Fig. 6A, Supplementary Table S7). The top different compounds were sugars, including sorbose, mannose, 2,7-anhydro-beta-sedoheptulose and fructose. The relative abundances of these compounds decreased when higher mucin was added to the BHI medium (Fig. 6A). This indicates that mucin addition may enhance carbon metabolism of A. muciniphila. Mannose is present in mucin and can be used for $\mathrm{N}$-glycan biosynthesis. However, mannosidase was not annotated in the genome of $A$. muciniphila. It is still unknown how mannose is produced and the most important is that bacteria need a balanced carbon-nitrogen ratio in the nutrient-rich conditions $s^{38}$. These significantly different metabolites were annotated in KEGG database and related pathways were enriched. Compared with the BHI group, addition of $0.05 \%$ mucin upregulated the pathways related to mucin degradation (beta-alanine, glycine, serine and threonine metabolism) and energy metabolism (phosphotransferase system, PTS) $(\mathrm{P}<0.05$, Fig. $7 \mathrm{~A})$. When the mucin addition increased to $0.5 \%$, more pathways were significantly enriched, which are related to mucin degradation (beta-alanine, tyrosine, arginine and proline metabolism, galactose metabolism, and amino sugar and nucleotide sugar metabolism), energy metabolism (PTS), and pantothenate and CoA biosynthesis (Fig. 7B,C). Common to each mucin was a proline-threonine-serine (PTS) domain and the PTS domain was the site of extensive O-glycosylation with carbohydrates accounting for up to $80 \%$ of the total mucin mass ${ }^{39}$. It is likely that high concentration of mucin in the BHI medium could stimulate A. muciniphila to produce numerous glycoside hydrolases. First, the release of sialic acid from non-reducing ends is an initial step in the sequential degradation of mucins ${ }^{40}$. Second, the mucin pore glycans were exposed to further enzymatic degradation for other glycoside hydrolases ( $\beta$-hexosaminidase, $\beta$-galactosidase and $\alpha$-L-fucosidase etc.). Finally, the bacteria would enter the PTS pathway for further energy metabolism and promote cell growth ${ }^{41}$.

Metabolome analysis revealed that the relative abundance of glucose and fructose decreased as the mucin addition increased $(\mathrm{P}<0.05$, Fig. $6 \mathrm{~B})$. During the cultivation, the absolute content of glucose showed an increase from 0 to $9 \mathrm{~h}$, but it decreased greatly afterwards $(\mathrm{P}<0.05)$. The absolute content of fructose increased from 0 to $18 \mathrm{~h}$ and then declined $(\mathrm{P}<0.05)$. The addition of mucin to BHI medium appeared to accelerate the breakdown of glucose (Fig. 6B). This is because glucose plays an important role in energy metabolism and is the preferred energy source for bacteria. BHI medium contains both glucose and nitrogen source. The three cultures had significantly different relative abundances of phenylalanine, threonine, lysine, ornithine, asparagine, alanyl-alanine, glutamic acid, leucine and valine $(\mathrm{P}<0.05$, Fig. $6 \mathrm{C})$. In particular, the relative abundance of free threonine decreased as the mucin addition increased in the BHI medium, which probably explains the downregulation of threonine-related DEGs in the BM0.5 group (Supplementary Table S8). Free leucine and valine were highly abundant in the BM0.5 group $(\mathrm{P}<0.05)$, while other free amino acids were more abundant in the BHI-only group $(\mathrm{P}<0.05)$, which could be attributed to the downregulation of DEGs involved in leucine biosynthesis (isopropylmalate dehydrogenase; Amuc_0667, Amuc_0785) and mucin degradation in the BM0.5 group, indicating the existence of a similar mechanism for formation of special amino acids in A. muciniphila ${ }^{42,43}$. As shown above, the genes of mucin-degrading enzymes were upregulated by $0.5 \%$ mucin addition, but extracellular activities of these enzymes were lower. As an enzymatic product, $\mathrm{N}$-acetylneuraminic acid was highly abundant in the BM0.5 group, which may inhibit the production of other acid substances (Fig. 6A).

Mechanisms on how A. muciniphila responds to high mucin under integrative analysis of transcriptome and metabolome data. To establish a relationship between DEGs and metabolites, several genes and metabolic pathways were listed in Supplementary Table S9. Three pathways were significantly enriched in the BM0.5 group compared with the BHI or BM0.05 groups, that is, amino sugar and nucleotide sugar metab- 
A

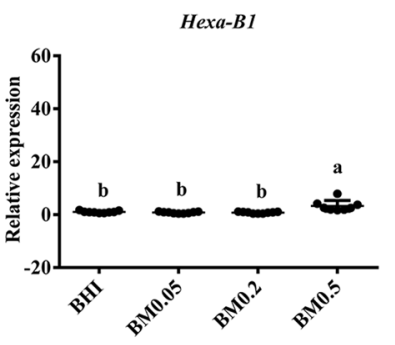

Hexa-B5

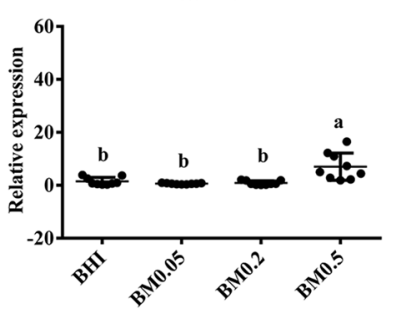

LacZ3

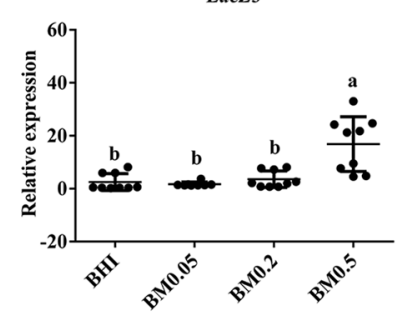

NaGlu1

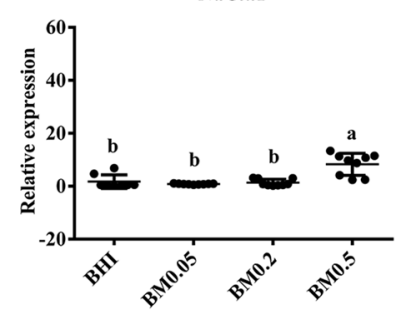

Hexa-B2

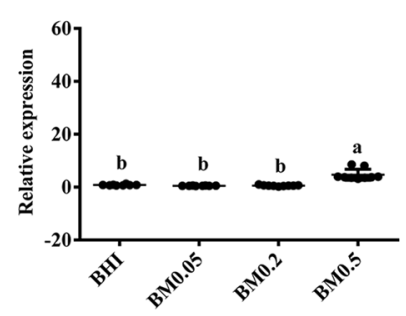

Hexa-B6

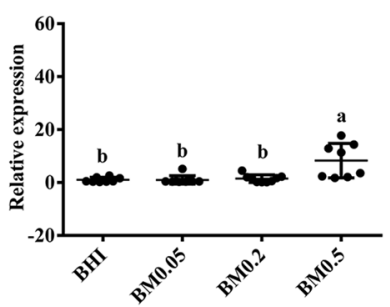

FucAl

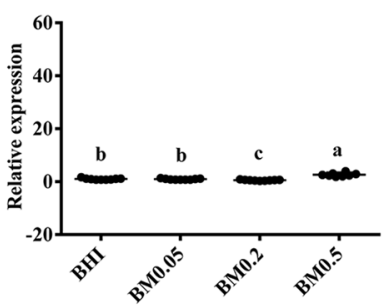

NaGlu2

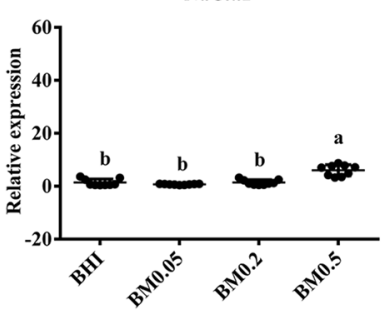

Hexa-B3

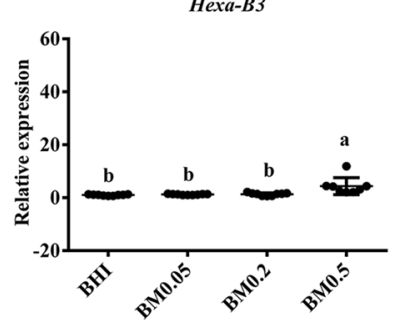

LacZI

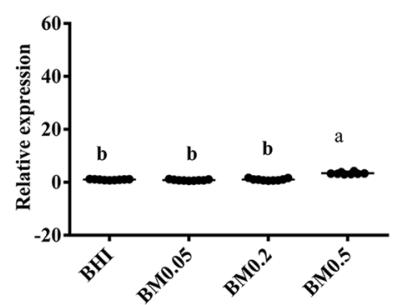

FucA2

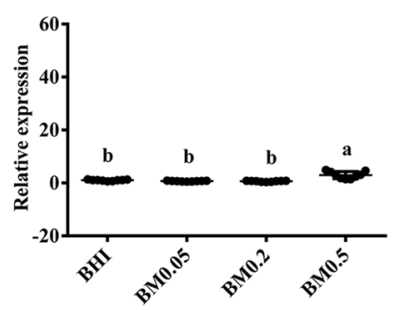

GalA

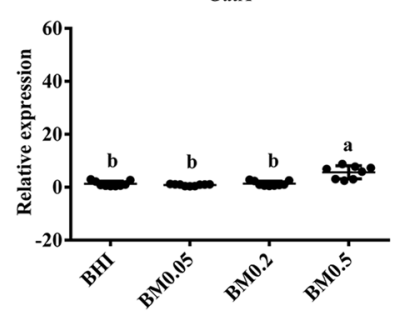

Hexa-B4

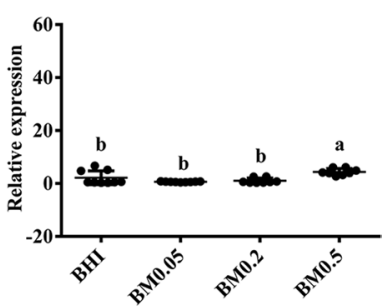

LacZ2

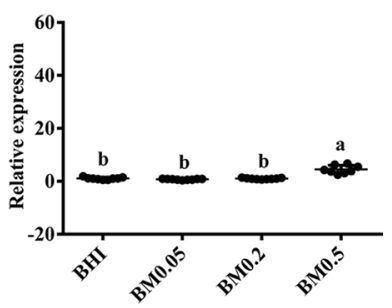

FucA3

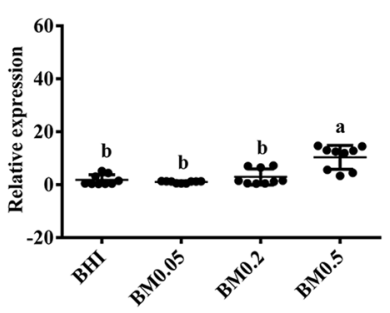

malZ

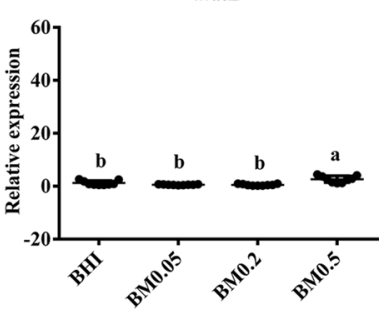

B

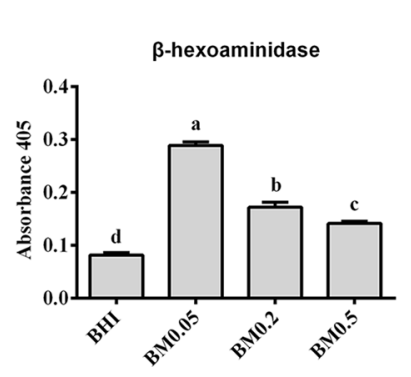

C

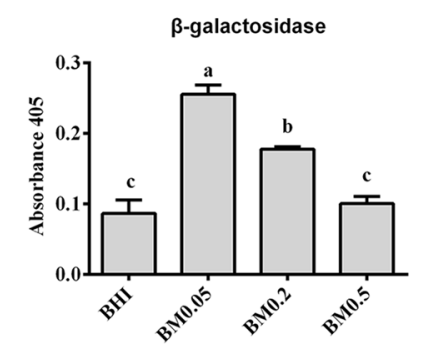

D

Figure 4. qRT-PCR and activities of mucin degrading enzymes. (A) qRT-PCR results; (B) and (C) Reactions were incubated with 4 -nitrophenyl- $\mathrm{N}$-acetyl- $\beta$-D-glucosaminide or 4 -nitrophenyl- $\beta$-D-galactopyranoside in PBS buffer $(50 \mathrm{mM}, \mathrm{pH} 7.0)$ for $1 \mathrm{~h}$ at $37^{\circ} \mathrm{C}$; (D) $\alpha$-L-fucosidase activities were measured by incubation with 2-chloro-4-nitrophenyl- $\alpha$-L-fucopyranoside in PBS buffer $(50 \mathrm{mM}, \mathrm{pH} 7.0)$ for $2.5 \mathrm{~h}$ at $37^{\circ} \mathrm{C}$. All these enzymatic reactions were stopped by adding $100 \mu \mathrm{l} 1 \mathrm{M} \mathrm{Na}_{2} \mathrm{CO}_{3}$ and the absorbance was read at $405 \mathrm{~nm}$. "a" "b" " $c$ " and " $\mathrm{d}$ " indicate significant differences in enzyme activities.

olism, galactose metabolism and ABC transporters. Mucin contains a large amount of amino sugars (GlcNAc and GalNAc), indicating that A. muciniphila significantly accelerated its cell growth in the BM0.5 group. 


\section{Cell shape regulation and cell division}

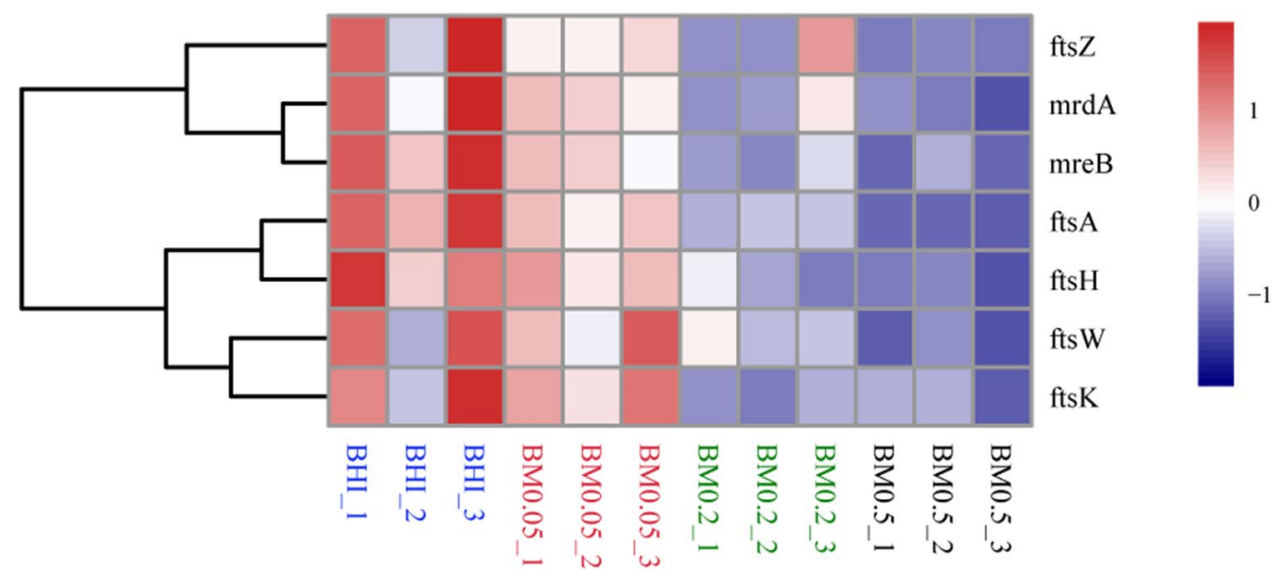

Figure 5. Heatmap analysis of genes related to cell morphology.

In the amino sugar and nucleotide sugar metabolism pathway, genes encoding amino sugar degrading enzymes, e.g., $\mathrm{N}$-acetylneuraminatelyase, hexosaminidase and glucosamine-6-phosphate deaminase were enriched. Glucosamine-6-phosphate deaminase catalyzes the conversion of glucosamine 6-phosphate to fructose 6-phosphate. The gene encoding UTP-glucose-1-phosphate uridylyltransferase was downregulated, which catalyzes the synthesis of UDP-glucose from UTP and D-glucose 1-phosphate. Mannose-1-phosphateguanylyl transferase catalyzes the conversion of D-mannose 1-phosphate to GDP-mannose. In the galactose metabolism pathway, genes encoding alpha-galactosidase and beta-galactosidase were upregulated, while genes encoding galactokinase were downregulated. This might be related to the decreasing galactinol when the mucin addition increased from 0 to $0.5 \%$, which is a donor of galactose. $\mathrm{ABC}$ transporters are widely found in microorganisms and play an important role in nutrient intake $e^{44}$.

Except for the above three common metabolic pathways, other four specific metabolic pathways were identified in the BM0.5 group compared with the BHI group (Supplementary Table S10), that is, pyrimidine metabolism pathway, purine metabolism pathway, pantothenate and CoA biosynthesis pathway, and arginine and proline metabolism pathway. The first three pathways are involved in cell growth. In the fourth pathway, the downregulated gene encoding pyrroline-5-carboxylate reductase is related to proline metabolism ${ }^{45}$.

Based on the above findings, we proposed a mechanism on how A. muciniphila responded to mucin addition in a BHI medium by combining the existing pathways of $A$. muciniphila in the KEGG database with the achieved omics data in the present study (Fig. 8). High mucin addition induced A. muciniphila to overexpress fucosidase, $\beta$-galactosidase, hexosaminidase to degrade mucins into fucose, $\mathrm{N}$-acetylgalactosamine and $\mathrm{N}$-acetylglucosamine. The resulting oligosaccharides could be broken down into monosaccharides, which eventually entered the glycolysis pathway to produce ATP or intermediates (e.g., pyruvate and alpha-ketoglutarate). These intermediates would go in different ways. For example, some intermediates can upregulate ketol-acid reductoisomerase (ilvC, Amuc_1178) and L-aspartate oxidase (nadB, Amuc_1079), while other intermediates may downregulate pyruvate dehydrogenase, dihydrodipicolinate reductase (dapB, Amuc_0257) and diaminopimelate dehydrogenase (ddh, Amuc_0581). Mucin addition upregulated succinyl-CoA ligase (sucC, Amuc_1713), but downregulated aconitate hydratase (acnA, Amuc_0904). These could reflect the regulations of ATP production.

In conclusion, mucin was found to be a good stimulus to proliferate this bacterium by upregulating expression of genes encoding glycosidase hydrolase, and cell shape and division. Mucin was also a good source of carbohydrates and amino acids for A. muciniphila to change its metabolic properties. High mucin addition upregulated genes involving nutrient uptake, cell growth, and cell morphology, probably by regulating ATP production. In a nutrient-enriched culture system, A. muciniphila showed a mucin-dependent growth by degrading mucin to maintain energy balance.

\section{Methods}

Bacterial strain and culture preparation. A. muciniphila (strain DSM 22959), which was stored in an anaerobic tube with $30 \%$ glycerol at $-80^{\circ} \mathrm{C}$, was obtained from DMSZ (German Collection of Microorganisms and Cell Culture) ${ }^{46}$. It was reactivated twice in BHI broth (Haibo, Qingdao, China, containing $10 \mathrm{~g} / \mathrm{L}$ tryptone, $17.5 \mathrm{~g} / \mathrm{L}$ beef heart powder, $2.0 \mathrm{~g} / \mathrm{L}$ glucose, $5 \mathrm{~g} / \mathrm{L} \mathrm{NaCl}$ and $2.5 \mathrm{~g} / \mathrm{L} \mathrm{Na}_{2} \mathrm{HPO}_{4} \cdot 12 \mathrm{H}_{2} \mathrm{O}$ ), which was supplemented with $0.2 \%(\mathrm{~m} / \mathrm{v})$ porcine gastric mucin (Type III, Sigma-Aldrich, St, Louis, MO) for $48 \mathrm{~h}$ at $37^{\circ} \mathrm{C}$. Porcine gastric mucin (type III; Sigma-Aldrich, St. Louis, MO) was purified by ethanol precipitation as described previously ${ }^{47}$. Briefly, $10 \mathrm{~g}$ of mucin was stirred for $24 \mathrm{~h}$ at $4{ }^{\circ} \mathrm{C}$ in $500 \mathrm{~mL}$ of $0.02 \mathrm{M}$ phosphate buffer (pH 7.4) containing $0.1 \mathrm{M}$ $\mathrm{NaCl}$ and a few drops of toluene. After the first hour, the $\mathrm{pH}$ was adjusted to 7.2 with $2 \mathrm{M} \mathrm{NaOH}$. After centrifugation at $10,000 \mathrm{~g}$, the supernatant was cooled to $0{ }^{\circ} \mathrm{C}$ and cooled ethanol $\left(4^{\circ} \mathrm{C}\right)$ was added to a final concentration of $60 \%(\mathrm{~V} / \mathrm{V})$. The resulting precipitate was suspended in $0.1 \mathrm{M} \mathrm{NaCl}$ and precipitated in $60 \%$ ethanol $(\mathrm{V} / \mathrm{V})$ at $4{ }^{\circ} \mathrm{C}$. The total precipitate was washed with $100 \%$ ethanol and dissolved in distilled water. Then, the mucin solution was dialyzed against distilled water for $24 \mathrm{~h}$ at $4{ }^{\circ} \mathrm{C}$. The dialyzed mucin solution was then lyophilized, 


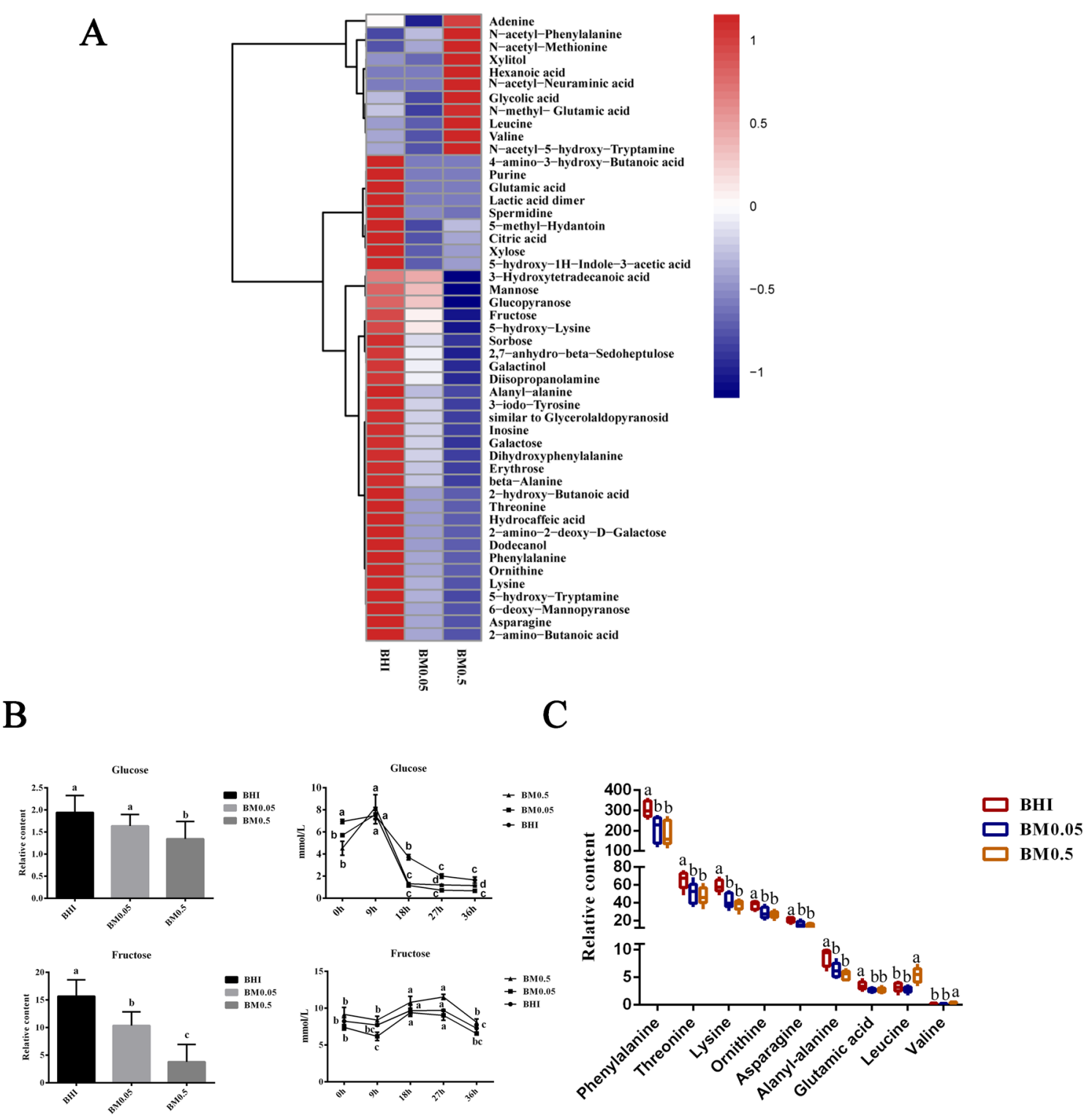

Figure 6. Metabolites in mucin-containing cultures (A) Heatmap of metabolites; (B) glucose and fructose varying in BHI, BM0.05 and BM0.5 cultures. "a" "b" "c" and "d" indicate significant differences among different time points. $(\mathbf{C})$ Relative contents of amino acids $(\mathrm{P}<0.05)$.

dissolved in distilled water, and autoclaved before use. Then the optical density was measured under a microplate reader at $600 \mathrm{~nm}$ (Infinite M200, Tecan Group, Männedorf, Switzerland) and an inoculum with OD value of 0.15 was taken to prepare a solution of $10^{8} \mathrm{CFU} / \mathrm{mL}$. Then the inoculum was incubated with $0 \%$ (control), $0.05 \%, 0.2 \%$ or $0.5 \%$ porcine gastric mucin (m/v, Sigma-Aldrich, St, Louis, $\mathrm{MO}$ ) in a BHI medium (designated as BHI, BM0.05, BM0.2 and BM0.5, pH 7.0-7.4) respectively. There were three replicates each group at each time point, and all the samples were prepared with the same batch of mucin. All the medium solutions need to be preheated and an aliquot of $0.1 \%$ resazurin oxygen indicator $(0.1 \mathrm{~mL})$ was added to each medium. Then, $0.1 \mathrm{~g}$ $\mathrm{L}$-cysteine hydrochloride was added under anaerobic condition and $9.5 \mathrm{~mL}$ medium was quickly dispensed into $30 \mathrm{~mL}$ serum bottles sealed with butyl-rubber stoppers. The medium was sterilized by autoclaving for $15 \mathrm{~min}$ at $121^{\circ} \mathrm{C}$. Incubations were performed in serum bottles sealed with butylrubber stoppers at $37^{\circ} \mathrm{C}$ under anaerobic conditions provided by a gas phase of $182 \mathrm{kPa}(1.8 \mathrm{~atm}) \mathrm{N}_{2}-\mathrm{CO}_{2}$. The culture volume was $10 \mathrm{ml}$ and the cultures were inoculated with $5 \times 10^{7}$ bacterial cells from bacterial cultures. Negative controls comprised of a series of mucin media that was not inoculated. In addition, mucin medium was used to culture A. muciniphila according to the procedure of Derrien et al. ${ }^{3}$. The basic medium was prepared by dissolving $0.4 \mathrm{~g} \mathrm{KH}_{2} \mathrm{PO}_{4}, 0.76 \mathrm{~g}$ $\mathrm{Na}_{2} \mathrm{HPO}_{4} \cdot 12 \mathrm{H}_{2} \mathrm{O}, 0.3 \mathrm{~g} \mathrm{NaCl}, 0.3 \mathrm{~g} \mathrm{NH}_{4} \mathrm{Cl}, 0.1 \mathrm{~g} \mathrm{MgCl}_{2} \cdot 6 \mathrm{H}_{2} \mathrm{O}, 0.11 \mathrm{~g} \mathrm{CaCl}_{2}, 4 \mathrm{~g} \mathrm{NaHCO}_{3}, 0.25 \mathrm{~g} \mathrm{Na}_{2} \mathrm{~S}_{7} \cdot 9 \mathrm{H}_{2} \mathrm{O}$, $0.5 \mathrm{mg}$ resazurin and $5 \mathrm{~g}$ porcine gastric mucin in double distilled water, and mixing $1 \mathrm{~mL}$ acid trace element solution, $1 \mathrm{~mL}$ alkaline trace element solution and $1 \mathrm{~mL}$ vitamin solution, and finally making up to $1000 \mathrm{~mL}$ with double distilled water. Acid trace element solution was made up by dissolving $1.49 \mathrm{~g} \mathrm{FeCl}_{2} \cdot 4 \mathrm{H}_{2} \mathrm{O}, 0.06 \mathrm{~g} \mathrm{H}_{3} \mathrm{BO}_{3}$, $0.07 \mathrm{~g} \mathrm{ZnCl}_{2}, 0.02 \mathrm{~g} \mathrm{CuCl}_{2} \cdot 2 \mathrm{H}_{2} \mathrm{O}, 0.06 \mathrm{~g} \mathrm{MnCl}_{2}, 0.12 \mathrm{~g} \mathrm{CoCl}_{2} \cdot 6 \mathrm{H}_{2} \mathrm{O}$, and $0.02 \mathrm{~g} \mathrm{NiCl}_{2} \cdot 6 \mathrm{H}_{2} \mathrm{O}$ in double distilled 
A

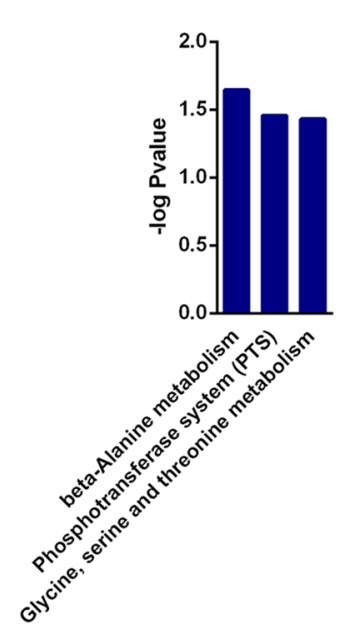

B

C
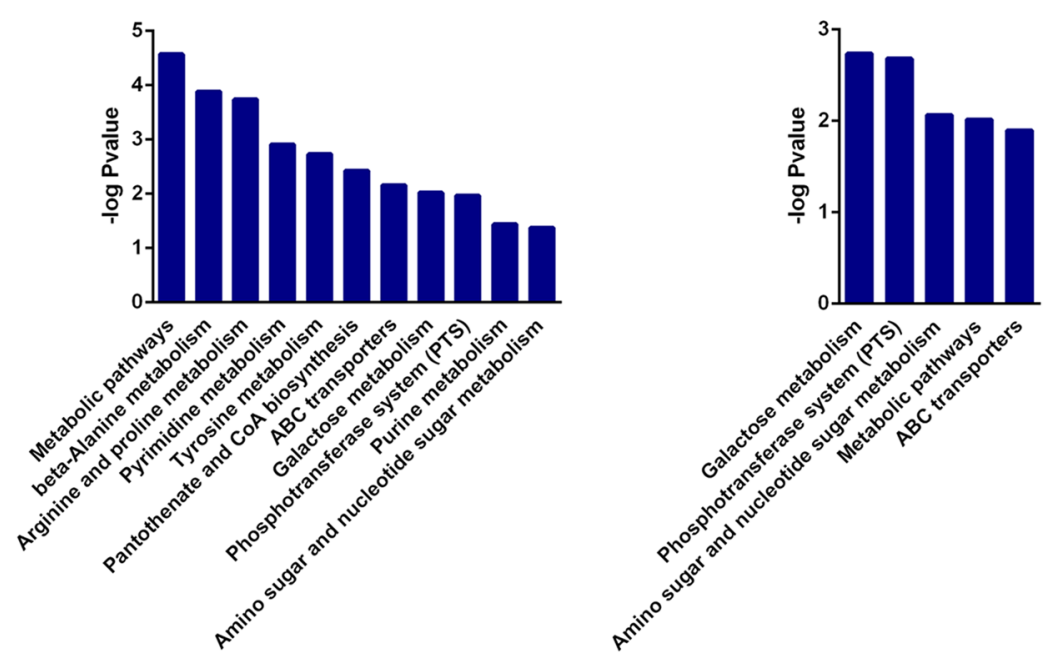

Figure 7. Enrichment of metabolites $(\mathrm{P}<0.05$, Fold change $>1)$ in KEGG database. (A-C) Pathways enriched in the KEGG database between BM0.05 and BHI groups, between BM0.5 and BHI groups, and between BM0.5 and BM0.05 groups, respectively. All the models were analyzed by the OmicsBean.

water and $4.2 \mathrm{~mL}$ concentrated $\mathrm{HCl}$ was added and the final volume was $1000 \mathrm{~mL}$. Alkaline trace element solution was prepared by dissolving $0.02 \mathrm{~g} \mathrm{Na}_{2} \mathrm{SeO}_{3}, 0.03 \mathrm{~g} \mathrm{Na}_{2} \mathrm{WO}_{4} \cdot 2 \mathrm{H}_{2} \mathrm{O}, 0.02 \mathrm{~g} \mathrm{Na}_{2} \mathrm{MoO}_{4} \cdot 2 \mathrm{H}_{2} \mathrm{O}$ and $0.4 \mathrm{~g} \mathrm{NaOH}$ in $1000 \mathrm{~mL}$ double distilled water. Vitamin solution was prepared by dissolving $20 \mathrm{mg}$ biotin, $200 \mathrm{mg}$ niacin, $500 \mathrm{mg}$ pyridoxine, $100 \mathrm{mg}$ riboflavin, $200 \mathrm{mg}$ thiamine, $100 \mathrm{mg}$ cyanocobalamin, $100 \mathrm{mg}$ p-aminobenzoic acid and $100 \mathrm{mg}$ pantothenic acid in $1000 \mathrm{~mL}$ double distilled water. For solid plate culture, the normal saline for a dilution of bacterial culture needed to be preheated and $1 \%(\mathrm{~V} / \mathrm{V})$ of $0.1 \%$ resazurin oxygen indicator was added. Then, $1 \%(\mathrm{~V} / \mathrm{V}) \mathrm{L}$-cysteine hydrochloride was added under anaerobic condition and $4.5 \mathrm{~mL}$ saline solution was quickly dispensed into $10 \mathrm{~mL}$ anaerobic tubes sealed with butyl-rubber stoppers. All the normal saline was sterilized by autoclaving for $15 \mathrm{~min}$ at $121^{\circ} \mathrm{C}$ and $0.5 \mathrm{ml}$ bacterial culture was injected into an anaerobic tube for serial dilution. Selected the appropriate dilution of bacterium for cultivation and counted at $37^{\circ} \mathrm{C}$ in an anaerobic workstation (Bugbox M, Ruskinn Technology, Wales, UK).

Scanning and transmission electron microscopy. Scanning electron microscopy was performed to visualize the shape of $A$. muciniphila as described by Bautista-Rosales et al. ${ }^{4}$. Images were obtained with a Hitachi S-3000N (Hitachi, Chiyoda, Tokyo, Japan). Transmission electron microscopy was applied to visualize ultrastructural changes in the microbial cells, the method was followed by Marrie et al. ${ }^{49}$. Finally, microbial structures were observed under a Hitachi h-7650 transmission electron microscope (Hitachi, Chiyoda, Tokyo, Japan).

RNA isolation and analysis. A typical growth curve of a bacterium comprises of lag phase, log or exponential phase, stationary phase, death phase and/or prolonged stationary phase. The time from the early log phase to the early stationary phase, and the abundance and metabolites of a bacterium at the early stationary phase may reflect the growth performance of the bacterium in a specific medium ${ }^{50}$. Thus, RNA was extracted from the cultures at the time points of the maximal abundance of the bacterium (early stationary phase), i.e., $35 \mathrm{~h}$ for BHI, $31 \mathrm{~h}$ for BM0.05, $30 \mathrm{~h}$ for BM0.2, and $28 \mathrm{~h}$ for BM0.5. RNA extraction were treated with the TRIzol reagent (Thermo Fisher Scientific, Carlsbad, CA) according to the manufacturer's protocols. The RNA quality was checked by $1 \%$ agarose gel electrophoresis, and the quantity and integrity were measured using a NanoDrop ND-2000 spectrophotometer (Thermo Fisher Scientific, Carlsbad, CA) and an Agilent 2100 Bioanalyzer (Agilent Technologies, Santa Clara, CA). Only samples with a RIN (RNA integrity number) score greater than 6.0 were used for library construction ${ }^{51}$.

A total amount of $3 \mu \mathrm{g}$ RNA per sample was taken. Ribosomal RNA was removed using a Ribo-Zero rRNA removal kit (Illumina, San Diego, CA). First strand cDNA was synthesized using random hexamer primer and M-MuLV Reverse Transcriptase (RNaseH-). Second strand cDNA synthesis was subsequently performed using DNA Polymerase I and RNase H. In the reaction buffer, dNTPs with dTTP were replaced by dUTP. Remaining overhangs were converted into blunt ends via exonuclease/polymerase activities. After adenylation of $3^{\prime}$ ends of DNA fragments, NEBNext Adaptor with hairpin loop structure was ligated to prepare for hybridization. In order to select cDNA fragments of preferentially 150-200 bp in length, the library fragments were purified with AMPure XP system (Beckman Coulter, Beverly, MA). Then $3 \mu$ L USER Enzyme (New England Biolabs, Ipswich, MA) was used with size-selected, adaptor-ligated cDNA at $37^{\circ} \mathrm{C}$ for $15 \min$ followed by 5 min at $95^{\circ} \mathrm{C}$. Then PCR was done with Phusion High-Fidelity DNA polymerase (New England Biolabs, Ipswich, MA). Finally, products were purified (AMPure XP system) and library quality was assessed on an Agilent Bioanalyzer 2100 system 


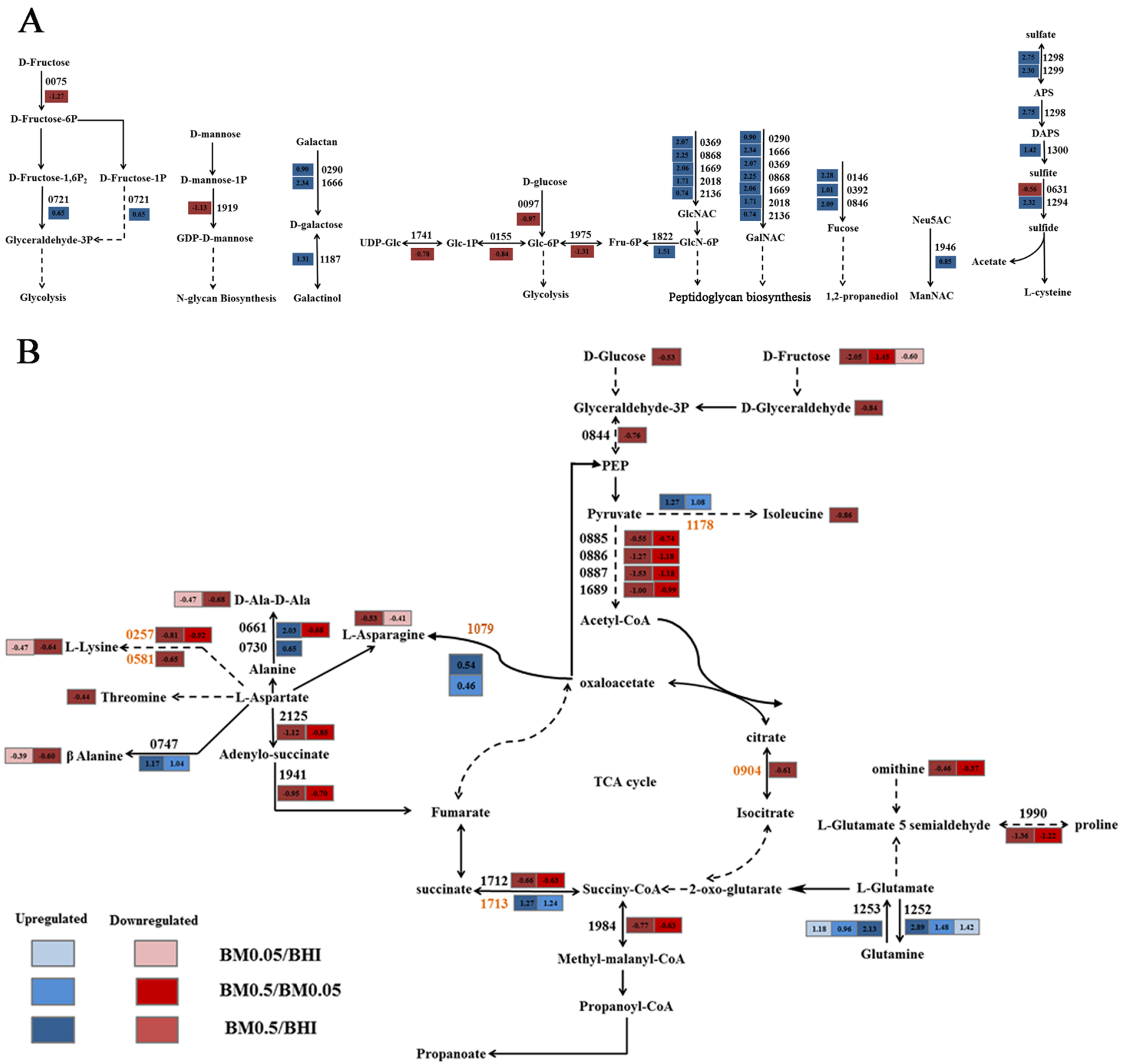

Figure 8. Proposed mechanism of mucin regulating the growth of $A$. muciniphila. (A) Pathways regulating the growth of A. muciniphila in BM0.5 group. (B) Pathway related to mucin-dependent metabolism in $A$. muciniphila. The proposed metabolic pathways were based on Swiss-Prot and KEGG databases. Blue boxes represent upregulated genes or metabolites, and red boxes represent downregulated genes or metabolites. The figures in the boxes represent $\log _{2}$ fold_changes between BM0.5 and BHI groups. The number on arrows represents the number of DEGs in KEGG database (For example: 0146 represent amu: Amuc_0146). Straight arrows indicate the direct effect between two substances. Dotted arrows indicate multiple steps between two substances. The proposed metabolic pathways are based on KEGG database (http://www.kegg.jp/kegg/kegg1. html).

(Agilent, Santa Clara, CA). A reference transcriptome was generated using A. muciniphila under BHI medium. Twelve libraries were constructed from the four different groups, and raw reads were obtained from HiSeq 2500 sequencing system (Illumina, San Diego, CA). Clean data were obtained by removing reads containing adapter and ploy-N, and low quality reads from raw data. At the same time, Q20, Q30 and GC content in the clean data were calculated. All the downstream analyses were based on clean data with high quality.

Clean data was transformed by $\log 10$ arithmetic and PCA was performed on the transformed data using DESeq2 (R package, version 1.30.0). In addition, the same R package was applied to acquire Venn diagrams to reflect the number of DEGs and heatmap to visualize the gene expression patterns. GO enrichment analysis of DEGs was performed by the GOseq R package (version 1.30.0, https://www.r-project.org/) and KOBAS (version 2.0, http://kobas.cbi.pku.edu.cn/) was applied to test the significant enrichment of DEGs in KEGG pathways. GO 
terms and KEGG pathways with $P$ values smaller than 0.05 were considered significantly enriched. Raw sequencing data can be accessed through SRA accession no. PRJNA559704. The genome sequence of $A$. muciniphila was deposited in GenBank under the accession number CP042830.

Quantitative RT-PCR analysis. Several genes involving mucin degradation were selected for quantitative reverse transcription-PCR (qRT-PCR) to validate if these enzymes were highly expressed, and each group had three replicates. Total RNA was isolated as described above and the CDNA was synthesized using the PrimeScript RT Master Mix kit (Takara, Kusatsu, Shiga, Japan) following the manufacturer's protocol. qRT-PCR was carried out using the SYBR Green probe on a QuantStudio 6 Flex system (Applied Biosystems, Foster City, CA). The cycling conditions were set as follows: initial denaturation at $95^{\circ} \mathrm{C}$ for $30 \mathrm{~s}$, and then 40 amplification cycles of $95^{\circ} \mathrm{C}$ for $5 \mathrm{~s}$ and 60 for $34 \mathrm{~s}$. Melting curve analysis was used to validate the specificity of primers. Levels of target gene transcripts were calculated relative to the $16 \mathrm{~S}$ rRNA using the $2^{-\Delta \Delta \mathrm{Ct}}$ method to normalize expression levels ${ }^{3}$. Primers are listed in supplemental materials Table S11.

Determination of extracellular glycoside hydrolase activity. The activities of $\beta$-hexosaminidase, $\beta$-galactosidase and $\alpha$-L-fucosidase were determined as described by Wendeler and Elhenawy et al. ${ }^{52,53}$. Briefly, $10 \mathrm{~mL}$ of bacterial cultures at the time points of the maximal abundance of the bacterium were centrifuged at $10,000 \times g$ for $10 \mathrm{~min}$ and the supernatant was passed through a $0.22-\mu \mathrm{m}$ Millipore membrane. Twenty microliters of the filtrate was incubated with $80 \mu \mathrm{L} 50 \mathrm{mM}$ phosphate buffer solution (PBS, pH7.0). To determine the activities of $\beta$-hexosaminidase, $\beta$-galactosidase and $\alpha$-L-fucosidase, appropriate amount of 4 -nitrophenyl- $\mathrm{N}$-acetyl$\beta$-D-glucosaminide, 4-nitrophenyl- $\beta$-D-galactopyranoside or 2 -chloro-4-nitrophenyl- $\alpha$-L-fucopyranoside was added, respectively, as substrates to a final concentration of $1 \mathrm{mmol} / \mathrm{L}$. The $\beta$-hexosaminidase and $\beta$-galactosidase involving reactions were done at $37^{\circ} \mathrm{C}$ for $1 \mathrm{~h}$. The $\alpha$-L-fucosidase involving reaction was done at $37^{\circ} \mathrm{C}$ for $2.5 \mathrm{~h}$. All these enzymatic reactions were stopped by adding $100 \mu \mathrm{L} 1 \mathrm{M} \mathrm{Na}_{2} \mathrm{CO}_{3}$ and the absorbance was read at $405 \mathrm{~nm}$. Each group had three replicates.

Metabolome profiling by gas chromatography-mass spectrometry (GC-MS). The cultures were taken at the time points of the maximal abundance of the bacterium, i.e., $35 \mathrm{~h}$ for BHI, $31 \mathrm{~h}$ for BM0.05, $30 \mathrm{~h}$ for $\mathrm{BM} 0.2$, and $28 \mathrm{~h}$ for BM0.5 ( $\mathrm{n}=5 \mathrm{each}$ ) for non-targeted metabolomic analysis as described in Mourão et al. ${ }^{54}$. GC-MS analysis was performed by Moros et al. ${ }^{55}$. Metabolites were identified by analysis of variance using the SAS software (version 8.0.1, Cary, NC). Significant differences were declared at the level of 0.05. KEGG and functional enrichment analyses were performed via omicsbean (http://www.omicsbean.cn/). Orthogonal partial least multiplication discriminant analysis (OPLS-DA) was done under the SIMCA 14.1 program. The data of metabolome was deposited in iProX under the project id PXD024558.

Determination of glucose and fructose. To validate the ability of A. muciniphila to utilize monosaccharides, glucose and fructose concentrations in the culture were monitored during the growth of bacteria at five time points $(0 \mathrm{~h}, 9 \mathrm{~h}, 18 \mathrm{~h}, 27 \mathrm{~h}$ and $36 \mathrm{~h})$. Five milliliters of culture were ultrasonically treated with $200 \mathrm{~W}$ for $3 \mathrm{~s}$ with a burst of $10 \mathrm{~s}$. The treatment was repeated 100 times. The resulting mixture was heated in a $95^{\circ} \mathrm{C}$ water bath for $10 \mathrm{~min}$. The mixture was centrifuged at $1000 \times g$ at $4{ }^{\circ} \mathrm{C}$ for $10 \mathrm{~min}$. Glucose and fructose in the supernatant were quantified by commercial kits (No. F006 and No. A085, Jiancheng, Nanjing, China).

Statistical analysis. All the data were checked for normal distribution. The effect of mucin addition on the measured variables, culture absorbance, PCR data of 16 genes of interest, enzymatic activities, glucose and fructose concentrations, and amino acids was evaluated by one-way ANOVA and comparisons were performed by $\mathrm{t}$ test using the SAS software (version 8.0.1, Cary, NC). Significant differences were declared if $P$ value was less than 0.05 .

Received: 4 December 2020; Accepted: 12 April 2021

Published online: 27 April 2021

\section{References}

1. Shin, J. et al. Elucidation of Akkermansia muciniphila probiotic traits driven by mucin depletion. Front. Microbiol. 10, 1137 (2019).

2. Hedlund, B. P., Gosink, J. J. \& Staley, J. T. Verrucomicrobia div. nov., a new division of the bacteria containing three new species of Prosthecobacter. Antonie van Leeuwenhoek int. J. Gen. Mol. Microbiol. 72, 29-38 (1997).

3. Derrien, M., Vaughan, E. E., Plugge, C. M. \& de Vos, W. M. Akkermansia muciniphila gen. nov., sp. nov., a human intestinal mucindegrading bacterium. Int. J. Syst. Evol. Microbiol. 54, 1469-1476 (2004).

4. Derrien, M., Collado, M. C., Benamor, K., Salminen, S. \& de Vos, W. M. The mucin degrader Akkermansia muciniphila is an abundant resident of the human intestinal tract. Appl. Environ. Microbiol. 74, 1646-1648 (2008).

5. Collado, M. C., Derrien, M., Isolauri, E., de Vos, W. M. \& Salminen, S. Intestinal integrity and Akkermansia muciniphila, a mucindegrading member of the intestinal microbiota present in infants, adults, and the elderly. Appl. Environ. Microbiol. 73, 7767-7770 (2007).

6. Dao, M. C. et al. Akkermansia muciniphila and improved metabolic health during a dietary intervention in obesity: Relationship with gut microbiome richness and ecology. Gut 65, 426-436 (2015).

7. Hänninen, A. et al. Akkermansia muciniphila induces gut microbiota remodelling and controls islet autoimmunity in NOD mice. Gut 67, 1445-1453 (2018). 
8. Longhi, G., Sinderen, D. V., Ventura, M. \& Turroni, F. Microbiota and Cancer: The emerging beneficial role of Bifidobacteria in cancer immunotherapy. Front. Microbiol. 11, 575072 (2020).

9. Mangin, I. et al. Oral administration of viable Bifidobacterium pseudolongum strain Patronus modified colonic microbiota and increased mucus layer thickness in rat. FEMS Microbiol. Ecol. 94, fiyl77 (2018).

10. Chen, G. L. et al. Partners of patients with ulcerative colitis exhibit a biologically relevant dysbiosis in fecal microbial metacommunities. World J. Gastroenterol. 23, 4624-4631 (2017).

11. Zhai, Q., Feng, S., Arjan, N. \& Chen, W. A next generation probiotic, Akkermansia muciniphila. Crit. Rev. Food Sci. Nutr. 59, 1-10 (2018).

12. Atuma, C., Strugala, V., Allen, A. \& Holm, L. The adherent gastrointestinal mucus gellayer: Thickness and physical state in vivo. Am. J. Physiol. Gastrointest. Liver Physiol. 280, G922-G929 (2001).

13. Bansil, R. \& Turner, B. S. The biology of mucus: Composition, synthesis and organization. Adv. Drug Deliv. Rev. 124, 3-15 (2018).

14. Pullan, R. D. et al. Thickness of adherent mucus gel on colonic mucosa in humans and its relevance to colitis. Gut 35, 353-359 (1994).

15. Varum, F. J. O., Veiga, F., Sousa, J. S. \& Basit, A. W. Mucus thickness in the gastrointestinal tract of laboratory animals. J. Pharm. Pharmacol. 64, 218-227 (2012).

16. Van den Abbeele, P. et al. Arabinoxylans and inulin differentially modulate the mucosal and luminal gut microbiota and mucindegradation in humanized rats. Environ. Microbiol. 13, 2667-2680 (2011).

17. Schneeberger, M. et al. Akkermansia muciniphila inversely correlates with the onset of inflammation, altered adipose tissue metabolism and metabolic disorders during obesity in mice. Sci. Rep. 5, 16643 (2015).

18. Everard, A. et al. Cross-talk between Akkermansia muciniphila and intestinal epithelium controls diet-induced obesity. Proc. Natl. Acad. Sci. 110, 9066-9071 (2013).

19. Sovran, B. et al. Age-associated impairment of the mucus barrier function is associated with profound changes in microbiota and immunity. Sci. Rep. 9, 1437 (2019).

20. Authimoolam, S. P. \& Thomas, D. Biopolymeric mucin and synthetic polymer analogs: Their structure, function and role in biomedical applications. Polymers 8, 71 (2016).

21. Abodinar, A. et al. The physicochemical characterization of pepsin degraded pig gastric mucin. Int. J. Biol. Macromol. 87, 281-286 (2016).

22. Berry, D. et al. Host-compound foraging by intestinal microbiota revealed by single-cell stable isotope probing. Proc. Natl. Acad. Sci. USA. 110, 4720-4725 (2013).

23. van Passel, M. W. J. et al. The genome of Akkermansia muciniphila, a dedicated intestinal mucin degrader, and its use in exploring intestinal metagenomes. PLoS ONE 6, e16876 (2011).

24. Slámová, K., Bojarová, P., Petrásková, L. \& Křen, V. $\beta$-N-acetylhexosaminidase: What's in a name...? Biotechnol. Adv. 28, 682-693 (2013).

25. Wang, M. et al. Cloning, purification and biochemical characterization of two $\beta$-Nacetyl hexosaminidases from the mucin-degrading gut bacterium Akkermansia muciniphila. Carbohydr. Res. 457, 1-7 (2018).

26. Kosciow, K. \& Deppenmeier, U. Characterization of a phospholipid-regulated $\beta$-galactosidase from Akkermansia muciniphila involved in mucin degradation. MicrobiologyOpen 8, e00796 (2019).

27. Katayama, T., Fujita, K. \& Yamamoto, K. Novel bifidobacterial glycosidases acting on sugar chains of mucin glycoproteins. J. Biosci. Bioeng. 99, 457-465 (2005).

28. Shimada, Y. et al. $\alpha$-N-Acetylglucosaminidase from Bifidobacterium bifidum specifically hydrolyzes $\alpha$-linked $\mathrm{N}$-acetylglucosamine at nonreducing terminus of O-glycan on gastric mucin. Appl. Microbiol. Biotechnol. 99, 3941-3948 (2015).

29. Wakinaka, T. et al. Bifidobacterial a-galactosidase with unique carbohydrate-binding module specifically acts on blood group B antigen. Glycobiology 23, 232-240 (2013).

30. Lunina, N. A., Berezina, O. V., Veith, B., Zverlov, V. V. \& Velikodvorskaya, G. A. A Cluster of Thermotoga neapolitana genes involved in the degradation of starch and maltodextrins: The expression of the aglB and aglA genes in E. coli and the properties of the recombinant enzymes. Mol. Biol. 37, 686-694 (2003).

31. Ottman, N. et al. Genome-scale model and omics analysis of metabolic capacities of Akkermansia muciniphila reveal a preferential mucin-degrading lifestyle. Appl. Environ. Microbiol. 83, e01014-e1017 (2017).

32. Zhao, F. et al. Dietary protein sources differentially affect the growth of Akkermansia muciniphila and maintenance of the gut mucus barrier in mice. Mol. Nutr. Food Res. 63, 201900589 (2019).

33. Pudlo, N. A. et al. Symbiotic human gut bacteria with variable metabolic priorities for host mucosal glycans. MBio 6, e01282-15 (2015).

34. van der Ark, K. C. H. Metabolic characterization and viable delivery of Akkermansia muciniphila for its future application. PhD thesis (University of Wageningen, 2018).

35. Shively, J. M. et al. Bacterial and archaeal inclusions. In eLS. Ltd: Chichester (John Wiley \& Sons, 2011).

36. Janeček, Š, Svensson, B. \& MacGregor, E. A. a-Amylase: An enzyme specificity found in various families of glycoside hydrolases. Cell. Mol. Life Sci. 71, 1149-1170 (2014).

37. Klumpp, S., Zhang, Z. \& Hwa, H. Growth-rate dependent global effects on gene expression in bacteria. Cell 139, 1366-1375 (2009).

38. Schumacher, J., Wang, B., Bonatto, A. C. \& Buck, M. Synthetic transcription factors allow regulon wide control and shifting the nitrogen/carbon balance in bacteria. New Biotechnol. 31, S22-S22 (2014).

39. Sheehan, J. K. et al. Identification of molecular intermediates in the assembly pathway of the MUC5AC mucin. J. Biol. Chem. 279, 15698-15705 (2004).

40. Tailford, L. E., Crost, E. H., Kavanaugh, D. \& Juge, N. Mucin glycan foraging in the human gut microbiome. Front. Genet. 6, 81 (2015).

41. Kotrba, P., Inui, M. \& Yukawa, H. Bacterial phosphotransferase system (PTS) in carbohydrate uptake and control of carbon metabolism. J. Biosci. Bioeng. 92, 502-517 (2001).

42. Sycheva, E. V. et al. Overproduction of noncanonical amino acids by Escherichia coli cells. Microbiology 76, 712-718 (2007).

43. Bode, R. Valine inhibition of beta-isopropylmalate dehydrogenase takes part in the regulation of leucine biosynthesis in Candida maltose. Antonie Van Leeuwenhoek 60, 125-130 (1991).

44. Davidson, A. L., Dassa, E., Orelle, C. \& Chen, J. Structure, function, and evolution of bacterial ATP-binding cassette systems. Microbiol. Mol. Biol. Rev. 7, 317-364 (2008).

45. Meng, Z. et al. Purification, characterization and crystallization of pyrroline-5-carboxylate reductase from the hyperthermophilicarcheon Sulfolobus Solfataricus. Protein Expr. Purif. 64, 125-130 (2009).

46. Huang, K. et al. Biochemical characterisation of the neuraminidase pool of the human gut symbiont Akkermansia muciniphila. Carbohydr. Res. 415, 60-65 (2015).

47. Miller, R. S. \& Hoskins, L. C. Mucin degradation in human colon ecosystems. Fecal population densities of mucin-degrading bacteria estimated by a "most probable number" method. Gastroenterology 81, 759-765 (1981).

48. Bautista-Rosales, P. U. et al. Biocontrol action mechanisms of Cryptococcus laurentii on Colletotrichum gloeosporioides of mango. Crop Prot. 65, 194-201 (2014).

49. Marrie, T. J. \& Costerton, J. W. Scanning and transmission electron microscopy of in situ bacterial colonization of intravenous and intraarterial catheters. J. Clin. Microbiol. 19, 687 (1984). 
50. Robador, A. et al. Nanocalorimetry reveals the growth dynamics of Escherichia coli cells undergoing adaptive evolution during long-term stationary phase. Appl. Environ. Microbiol. 85, e00968-e1019 (2019).

51. Jahn, C. E., Charkowski, A. O. \& Willis, D. K. Evaluation of isolation methods and RNA integrity for bacterial RNA quantitation. J. Microbiol. Methods 75, 318-324 (2008).

52. Wendeler, M. \& Sandhoff, K. Hexosaminidase assays. Glycoconj. J. 26, 945-952 (2009).

53. Elhenawy, W., Debelyy, M. O. \& Feldman, M. F. Preferential packing of acidic glycosidases and proteases into bacteroides outer membrane vesicles. MBio 5, e00909-14 (2014).

54. Mourão, M. P. et al. Direct detection of Mycobacterium tuberculosis in sputum: A validation study using solid phase extraction-gas chromatography-mass spectrometry. J. Chromatogr. B Anal. Technol. Biomed. Life Sci. 986-987, 115-122 (2015).

55. Moros, G., Chatziioannou, A. C., Gika, H. G., Raikos, N. \& Theodoridis, G. Investigation of the derivatization conditions for GC-MS metabolomics of biological samples. Bioanalysis 9, 53-65 (2017).

\section{Acknowledgements}

This study was financially supported by grants from National Natural Science Foundation of China (no. 31530054), 10000 Talent Project, and Jiangsu Provincial Department of Education (PAPD). We sincerely thank Li Liu and Josef Voglmeir for providing the strain and guidance of bacterial culture.

\section{Author contributions}

C.L. designed the experiment. X.L., F.Z., H.L., D.Z. and Y.X. performed the experiment. X.L. and C.L. wrote the manuscript. All authors reviewed the manuscript.

\section{Competing interests}

The authors declare no competing interests.

\section{Additional information}

Supplementary Information The online version contains supplementary material available at https://doi.org/ 10.1038/s41598-021-88397-z.

Correspondence and requests for materials should be addressed to C.L.

Reprints and permissions information is available at www.nature.com/reprints.

Publisher's note Springer Nature remains neutral with regard to jurisdictional claims in published maps and institutional affiliations.

(c) (i) Open Access This article is licensed under a Creative Commons Attribution 4.0 International License, which permits use, sharing, adaptation, distribution and reproduction in any medium or format, as long as you give appropriate credit to the original author(s) and the source, provide a link to the Creative Commons licence, and indicate if changes were made. The images or other third party material in this article are included in the article's Creative Commons licence, unless indicated otherwise in a credit line to the material. If material is not included in the article's Creative Commons licence and your intended use is not permitted by statutory regulation or exceeds the permitted use, you will need to obtain permission directly from the copyright holder. To view a copy of this licence, visit http://creativecommons.org/licenses/by/4.0/.

(C) The Author(s) 2021 